\title{
Multi-modal diffuse optical techniques for breast cancer neoadjuvant chemotherapy monitoring (Conference Presentation)
}

Jeffrey M. Cochran David R. Busch

Han Y. Ban

Venkaiah C. Kavuri Martin J. Schweiger Simon R. Arridge Arjun G. Yodh 


\title{
Multi-modal diffuse optical techniques for breast cancer neoadjuvant chemotherapy monitoring (Conference Presentation)
}

\author{
Jeffrey M. Cochran, Univ. of Pennsylvania (United States); David R. Busch, The Children's \\ Hospital of Philadelphia (United States); Han Y. Ban, Venkaiah C. Kavuri, Univ. of \\ Pennsylvania (United States); Martin J. Schweiger, Simon R. Arridge, Univ. College London \\ (United Kingdom); Arjun G. Yodh, Univ. of Pennsylvania (United States)
}

\begin{abstract}
We present high spatial density, multi-modal, parallel-plate Diffuse Optical Tomography (DOT) imaging systems for the purpose of breast tumor detection. One hybrid instrument provides time domain (TD) and continuous wave (CW) DOT at 64 source fiber positions. The TD diffuse optical spectroscopy with PMT- detection produces lowresolution images of absolute tissue scattering and absorption while the spatially dense array of CCD-coupled detector fibers (108 detectors) provides higher-resolution CW images of relative tissue optical properties. Reconstruction of the tissue optical properties, along with total hemoglobin concentration and tissue oxygen saturation, is performed using the TOAST software suite. Comparison of the spatially-dense DOT images and MR images allows for a robust validation of DOT against an accepted clinical modality. Additionally, the structural information from co-registered MR images is used as a spatial prior to improve the quality of the functional optical images and provide more accurate quantification of the optical and hemodynamic properties of tumors. We also present an optical-only imaging system that provides frequency domain (FD) DOT at 209 source positions with full CCD detection and incorporates optical fringe projection profilometry to determine the breast boundary. This profilometry serves as a spatial constraint, improving the quality of the DOT reconstructions while retaining the benefits of an optical-only device. We present initial images from both human subjects and phantoms to display the utility of high spatial density data and multi-modal information in DOT reconstruction with the two systems.

View presentation recording on the SPIE Digital Library: http://dx.doi.org/10.1117/12.2251455.5371876645001
\end{abstract}

